\title{
Limited view thermoacoustic tomography
}

\author{
Yuan $\mathrm{Xu}$, Lihong $\mathrm{V}$. Wang \\ Biomedical Engineering Program, Texas A\&M University, College Station, TX 77843-3120, USA \\ http://oilab.tamu.edu
}

\begin{abstract}
Truncated conjugate gradient method was applied to study the limited view problem in thermoacoustic tomography, and the results were compared with those of modified backprojection method, which is the backprojection of the first order time derivative of acoustic signals. Our numerical simulations showed that there is complete data for a stable and perfect reconstruction in a 2-D $\pi$-view problem, where the least angle acquired by the detection curve is $\pi$ when viewed from the imaged region of interest. On the contrary, in a problem where the view is less than $\pi$, data is incomplete, and artifacts and quantitative errors were obvious in the reconstructed images. It was pointed out that the result after one iteration in truncated conjugate gradient method is equivalent to that of modified backprojection, which can restore the high frequency information of imaged objects. The low frequency information can be recovered significantly in the next ten iterations of truncated conjugate gradient method. Keywords - Thermoacoustic tomography, limited view, truncated conjugate gradient.
\end{abstract}

\section{INTRODUCTION}

In thermoacoustic tomography (TAT), the thermoacoustic signals from a tissue sample are collected to map the distribution of the radiation absorption within the sample. In the frequency domain, exact reconstruction algorithms for TAT have been implemented in planar, spherical, and cylindrical configurations with series expansion techniques [1-3]. In these algorithms, the thermoacoustic signals are assumed to be detected in a full view, which is from all directions around the imaged objects. A detection view is defined as the solid angle (3-D case) or angle (2-D case) acquired by a detection surface or line when viewed from the to-be-imaged objects. In TAT, a full view is $4 \pi$ solid angle in 3-D case or $2 \pi$ in 2-D case. This is an essential difference between thermoacoustic tomography and $\mathrm{X}$-ray tomography, in which a detection angle range of $\pi$ can produce complete data. However, in many applications of TAT, the signals cannot be collected from all directions. For example, the range of detection solid angle is at most $2 \pi$ in the breast imaging. A detection view of $2 \pi$ solid angle was shown to provide complete data under the approximation that the distance between the detector and the absorbing object is much larger than the dimension of the absorbing object [4].

On the other hand, one's physical intuition can sense that a detection of half-view, which is $2 \pi$ solid angle in 3-D case or $\pi$ in 2-D case, should be able to acquire sufficient data to reconstruct the complete image.

II. Methods
Assume microwave absorption $H(\mathbf{r}, t)=I_{0} \varphi(\mathbf{r}) \eta(t)$, where $I_{0}$ is a scaling factor proportional to the incident radiation intensity; $\varphi(\mathbf{r})$ describes the to-be-reconstructed microwave absorption properties of the medium at $\mathbf{r}$; and $\eta(t)$ describes the shape of the irradiating pulse. In the case of thermal confinement, we have [5]:

$p_{1}(\mathbf{r}, t)=\frac{\beta I_{0}}{4 \pi C} \frac{\partial}{\partial t} \iint_{|=| \mathbf{r}-\mathbf{r}^{\prime} \mid / v_{s}} \frac{\varphi\left(\mathbf{r}^{\prime}\right)}{\left|\mathbf{r}-\mathbf{r}^{\prime}\right|} d \mathbf{r}^{\prime}$

where $\bar{p}_{1}(\mathbf{r}, k)=\bar{p}(\mathbf{r}, k) / \bar{\eta}(k), \bar{p}$ and $\bar{\eta}$ are the Fourier transforms of pressure $p$ and $\eta$ with respect to time respectively; $v_{s}$ is the acoustic speed; $C$ is the specific heat; and $\beta$ is the coefficient of volume thermal expansion. The physical meaning of this equation is that, in an acoustically homogenous medium, the pressure $p_{1}$ at a spatial point $r$ and time $t$ is proportional to the first-order time derivative of the integration of the absorbed microwave energy over a spherical surface (circle in 2-D case). The spherical surface is centered at $r$ and the radius is $t_{s}$.

It can be seen from Eq. (1) that $p_{1}(\mathbf{r}, t)$ can be obtained from $\varphi\left(\mathbf{r}^{\prime}\right)$ after applying two linear operations to it: one is the integration over the sphere or circle in the object space, $\mathrm{R}$; the other is the differentiation over $t, \mathrm{D}_{t} . \mathrm{R}$ is similar to Radon transform except that the integration is over a sphere or circle rather than a plane or line as in Radon transform. We adopted the Truncated conjugate gradient (TCG) method to solve the linear system in our study, because there is a close relation between backprojection algorithms and TCG. In principle, all the approximate reconstruction algorithms mentioned in the introductory section are backprojection type. It can be showed that the result of TCG after one iteration is equivalent to that of the modified backprojection algorithm except a constant factor.

Quinto [6] pointed out what is visible singularity in X-ray transform. However, it is found that the direct extension of Quinto's results to TAT agrees quite well with our numerical simulation results. One of Quinto's results is that a singularity cannot be reconstructed stably unless an integral plane or line is tangent to that singularity. After applying his theory to TAT, it is straightforward to see that a half-view will provide complete data to reconstruct all the singularities.

The thermoacoustic imaging of several tumors in limited views was numerically simulated; Fig. 1 shows the diagrams of the tumors in a $\pi$-view and a sub- $\pi$-view (a view less than $\pi$ ) model. 
(a)

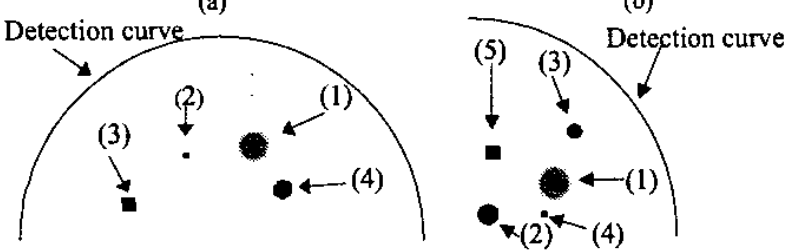

Fig. 1 shows the diagrams of tumors in a $\pi$-view and a sub- $\pi$-view model

\section{RESULTS AND DISCUSSION}

Fig. 2 shows the results for the $\pi$-view model [(a) and (b)] and sub- $\pi$-view model [(c) and (d)]. The left side images (a)

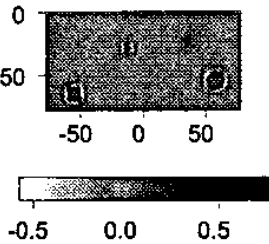

(c)

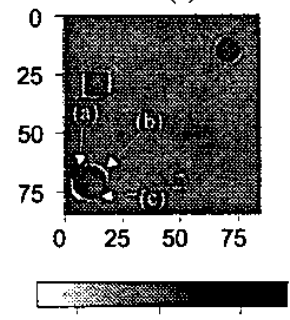

$\begin{array}{lll}-0.5 & 0.0 & 0.5\end{array}$ (b)

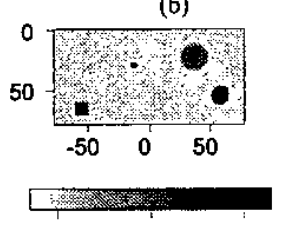

(d)

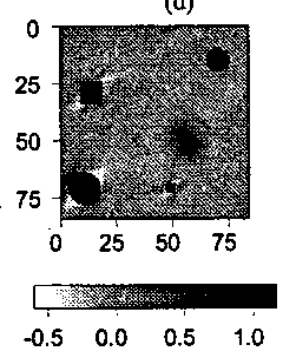

Fig. 2 shows that a $\pi$-view detection [(a) and (b)] provides complete data for reconstruction, and that a sub- $\pi$-view detection [(c) and (d)] provides incomplete data and therefore causes artifacts. The left side images are the results after one iteration, and the right side ones after 11 iterations. Comparison of the left ones with the right ones shows the improvement of TCG over modified backprojection. The unit of $\mathrm{x}$-axis and $\mathrm{y}$-axis is $\mathrm{mm}$.

are the results after one iteration, which are equivalent to those of the modified backprojection algorithm, and the right side ones after 11 iterations. It is clear to see the improvement of TCG over the modified backprojection by comparing the images in the two columns. For the $\pi$-view model, Fig. 2(a) shows that all the singularities are correctly reconstructed. The discontinuity in the first-order derivative at the boundary of tumor (1) was also recovered (it is much more clearly shown in the monitor than in paper). Compared Fig. 2(a) with Fig. 2(b), the defects in Fig. 2(a) can be readily determined. Firstly, there are obvious artifacts originating from each object, and these artifacts don't exist in a $2 \pi$-view model; secondly, tumor (1), which contained ore low frequency component than other tumors are much more poorly recovered; lastly, the value within each tumor is wrong. After ten more iterations, these defects are significantly remedied, as shown in Fig. 2(b). All the objects are reconstructed well quantitatively. On the contrary, Fig. 2(c) and (d) show that sub- $\pi$-view detection provides incomplete data for reconstruction. The artifacts and noise in images after one and 11 iterations in the sub- $\pi$-view model are much stronger than the corresponding ones in the half-view model.

\section{CONCLUSIONS}

Our numerical simulations showed that a $\pi$-view in a 2-D problem provides complete data for reconstruction. In a $\pi$ view problem, modified backprojection produced some artifacts and errors in the low frequency component of the images. The artifacts and errors can be reduced significantly after about ten iterations. On the contrary, in a sub- $\pi$-view problem, which has incomplete data, iterations improved the imaging quality, but artifacts and quantitative errors were still obvious. The direct extension of Quinto's results on visible and invisible singularity in X-ray transform to TAT agrees quite well with our numerical simulation results.

\section{ACKNOWLEDGMENTS}

This project was sponsored in part by the U.S. Army Medical Research and Materiel Command Grant No. DAMD17-00-1-0455, the National Institutes of Health Grants No. R01 CA71980 and No. R21 CA83760, the National Science Foudation Grant No. BES-9734491, and Texas Higher Education Coordinating Board Grant No. ARP 000512-0123-199

\section{REFERENCES}

[1] Y. Xu, D. Feng, L.-H. V. Wang, "Exact reconstruction algorithm by Fourier transform for thermoacoustic tomography in biological tissues," IEEE Trans. Med. Imag., accepted (2002).

[2] M. Xu and L.-H. V. Wang, "Pulsed-microwave-induced thermoacoustic tomography of biological tissues: reconstruction theory and experiments," IEEE Trans. Med. Imag., accepted (2002).

[3] Y. Xu, M. Xu, L. -H V. Wang, "Exact reconstruction algorithm in Fourier domain for thermoacoustic tomography in biological tissues: a cylindrical configuration," IEEE Trans. Med. Imag., accepted (2002).

[4] R. A. Kruger, P. Liu, Y. R. Fang, and C. R. Appledorn, "Photoacoustic ultrasound (PAUS)-reconstruction tomography," Med. Phys. vol. 22, pp. 1605-1609, 1995. [5]Y. Xu, L. -H V. Wang, "Effects of Acoustic Heterogeneity on Thermoacoustic Tomography in the Breast", submitted to IEEE Trans. Ultrason. Ferroel. Frequency Control.

[6] Quinto E. T., "Singularities of the X-ray transform and limited data tomography in $8^{2}$ and $8^{3}$ ", SIAM J. MATH. ANAL., vol. 24, pp. 1215 - 1225 (1993). 